\title{
Genomic Feature Physical Location
}

National Cancer Institute

\section{Source}

National Cancer Institute. Genomic Feature Physical Location. NCI Thesaurus. Code C64389.

The physical location on a specified chromosome of a feature or area of interest expressed as a base pair position. 